\title{
聚苯撑乙烯衍生物链间聚集与链内自聚集对光致发光的影响
}

\author{
张海全 ${ }^{1, *}$ 赵立成 ${ }^{1}$ 翟 静 $^{2}$ 刘文科 ${ }^{1}$ 李 影 ${ }^{1}$ 张 帅 ${ }^{1}$ \\ ( 1 燕山大学, 亚稳材料制备技术与科学国家重点实验室, 河北 秦皇岛 066004; \\ 2 燕山大学环境与化学工程学院, 河北 秦皇岛 066004)
}

\begin{abstract}
摘要: 利用 Gilch 路线合成了具有高度物理缠结的聚苯撑乙烯衍生物(聚 2,5-二-辛氧基苯撑乙烯(DOO-PPV)凝 胶及短链 DOO-PPV 粉末, 并利用傅里叶变换-红外光谱(FT-IR)及傅里叶变换-拉曼光谱(FT-Raman)进行了结构 表征, 结果表明, 凝胶与其短链 DOO-PPV 粉末具有相同的化学结构, 分子间不存在化学交联. 比较了短链 DOOPPV 粉末、凝胶、旋涂薄膜的紫外-可见(UV-Vis)光谱、光致发光 (PL) 光谱及 X 射线衍射(XRD)图谱, 发现在薄 膜、短链 DOO-PPV 粉末及凝胶的 PL 光谱中, 短波长发射峰强度与长波长发射峰强度的比值依次降低, 分别为 $3.24 、 1.10$ 和 0.47. XRD 分析表明, 凝胶中存在较多有序结构, 短链 DOO-PPV 粉末中分子有序结构较少, 薄膜中 分子有序性最低; 表明固态下 DOO-PPV 短波长的发射峰与链内自聚集相关, 而长波长发射峰与链间聚集有关.
\end{abstract}

关键词：聚苯撑乙烯; 链内自聚集; 链间聚集; 凝胶; 光致发光

中图分类号: 0641

\section{Influence of Interchain and Self Intrachain Aggregations on Photoluminescence of a Poly-Phenylenevinylene Derivative}

\author{
ZHANG Hai-Quan ${ }^{1, *} \quad$ ZHAO Li-Cheng ${ }^{1} \quad$ ZHAI Jing $^{2} \quad$ LIU Wen-Ke ${ }^{1} \quad$ LI Ying $^{1} \quad$ ZHANG Shuai $^{1}$ \\ ( ${ }^{I}$ State Key Laboratory of Metastable Materials Science and Technology, Yanshan University, Qinhuangdao 066004, \\ Hebei Province, P. R. China; ${ }^{2}$ College of Environmental and Chemical Engineering, Yanshan University,
} Qinhuangdao 066004, Hebei Province, P. R. China)

\begin{abstract}
A poly-phenylenevinylene derivative (poly (2,5-dioctyloxy)-p-phenylene vinylene) (DOO-PPV) with a high degree of physical crosslinking (designated the gelation of DOO-PPV) was synthesized and characterized. Analyses of the Fourier transform-infrared (FT-IR) and the Fourier transform-Raman (FT-Raman) spectra indicate that the chemical structure of the gelation of DOO-PPV is accordant with short chain linear molecule of DOO-PPV. Photoluminescence spectra of a thin film, short chain linear molecule, and solid gelation show that the ratio of the intensity at the short wavelength emitting band and at the long wavelength emitting band decreases gradually, the ratio is $3.24,1.10$ and 0.47 , respectively. Analysis of X-ray diffraction (XRD) indicated that the existence of a more oriented structure in the gelation of DOO-PPV compared with the short chain molecule and the thin film. This result suggests that interchain and self intrachain aggregations play an important role in the photoluminescence.
\end{abstract}

Key Words: Poly-phenylenevinylene; Aggregation of self intrachain; Aggregation of interchain; Gelation; Photoluminescence

聚苯撑乙烯(PPV)及其衍生物以其独特的光电性质, 广泛应用于发光二极管、太阳能电池等领

Received: December 9, 2008; Revised: February 13, 2009; Published on Web: April 9, 2009.

*Corresponding author. Email: hqzhang @ysu.edu.cn; Tel: +86335-8074791.

燕山大学博士基金(B141)资助项目

(C) Editorial office of Acta Physico-Chimica Sinica 
域 ${ }^{[1-3]}$. 聚合物半导体材料的光电性能不仅仅取决于 化学结构、分子量及分布等, 还强烈地依赖于固态下 材料的纳、微米级聚集态结构[4,5]. 尽管人们利用各种 思路探讨了聚合物半导体材料聚集态结构与发光性 质之间的关系, 但仍然存在一些值得讨论的问题. 例 如通过高斯拟合的聚 2-甲氧基-5-(2' - 乙基已氧基) 苯撑乙烯稀溶液的光致发光光谱存在三个特征发射 峰, 其峰值分别在 $550 、 580$ 和 $620 \mathrm{~nm}$ 左右, 其中 $550 \mathrm{~nm}$ 左右发射归属于单链内激子, 而 580 和 620 $\mathrm{nm}$ 发射归属于链间激子, 链间激子与共轭片断间 $\pi$ 电子云的叠加形式有关 ${ }^{[6-10]}$, 但共轭片段间 $\pi$ 电子 云叠加形式不仅仅包含不同分子间的 $\pi$ 电子云叠 加(由不同分子链间的聚集引起的, 这种聚集称之为 “链间聚集”), 同时也包含同一分子链内弯曲、折叠 等引起的 $\pi$ 电子云叠加(由同一分子链弯曲、折叠引 起的聚集, 本文称之为 “链内自聚集”), $\pi$-共轭聚合 物链内自聚集和链间聚集对光致发光产生怎样影响 还不甚清楚. 利用 Gilch 路线合成 PPV 衍生物的过 程中极易获得不溶的聚合物凝胶 ${ }^{[11,12]}$, 这种聚合物凝 胶被证明是由于分子间高度物理缠结所致. 聚苯撑 乙烯衍生物的主链具有高度刚性, 在聚合时具有较 快的链增长速度, 生成聚合物凝胶时同一分子链自 身来不及弯曲、折叠已被链间的缠结固定, 因此在这 种聚合物凝胶中可能存在不同长度的链间聚集有序 区域, 而链内自聚集较弱. 链内自聚集和链间聚集的 另一个极端情况是旋涂的聚合物薄膜, 烷氧基取代 的聚苯撑乙烯在四氢呋喃等良性溶剂中, 其分子链 容易弯曲、盘卷或折叠, 在旋涂成膜时, 溶剂快速挥 发使其高度的自聚集结构被保存下来, 同时由于分 子链自身的弯曲、扭曲等抑制了不同分子的链间聚 集, 因此对于薄聚合物膜来说可能存在较强的链内 自聚集和较弱的链间聚集. 因此比较凝胶和薄膜的 发光光谱能够进一步增加对链内自聚集(链自身的
弯曲、折叠和盘卷)及链间聚集(不同分子链间的相 互作用) 与其电子结构关系的理解. 聚-2,5-二-辛氧 基苯撑乙烯(DOO-PPV)是含有对称烷氧基取代的聚 苯撑乙烯衍生物, 其发光性质与其它双烷氧基取代 的聚苯撑乙烯基本相同. DOO-PPV 通常用来制备 短链长的双烷氧基取代的聚苯撑乙烯衍生物, 可获 得重复单元数小于 24 的可溶性的聚合物 ${ }^{[13]}$. 短链 DOO-PPV 分子在沉淀过程中链的缠结较困难, 故其 聚集态结构可能介于凝胶与薄膜之间. 因此在本文 中, 利用FT-IR、FT-Raman表征了DOO-PPV凝胶、短 链DOO-PPV粉末的结构, 比较凝胶、短链DOO-PPV 粉末和薄膜的PL光谱、UV-Vis光谱及相应的XRD图 谱, 探讨链内自聚集、链间聚集对其发光性能的影响.

\section{1 实 验}

\section{1 原 料}

对苯二酚(分析纯, 天津市巨星圣源化学试剂 厂), 1-溴-辛烷(化学纯, 南开大学精细化学实验厂), 乙腈(分析纯, 天津市科密欧化学试剂开发中心), 多 聚甲醛(化学纯, 上海天莲精细化工有限公司), 氢 溴酸的醋酸溶液(Acros 公司), 叔丁醇钾( $98 \%$, Acros 公司), 四氢呋喃、甲苯(分析纯, 天津市巨星圣源化 学试剂厂), 实验所用药品除四氢呋喃脱水精制, 其 余全部直接使用.

\section{2 仪器及测试}

E55×FRA106 型傅立叶变换红外光谱仪和傅里叶 变换激光拉曼光谱仪 (德国BRUKER公司), KBr压 片; RF-5301PC 苂光光谱仪(美国JY公司生产); UV3000 型紫外吸收光谱仪(日本 岛津); Rigaku D-max2500 型 X 射线衍射仪(日本 Rigaku 公司), $\mathrm{Cu} K_{\alpha}$ 辐 射源.

\section{3 短链的 DOO-PPV 粉末、凝胶和薄膜制备}

短链 DOO-PPV 粉末的合成路线见图 1. 首先

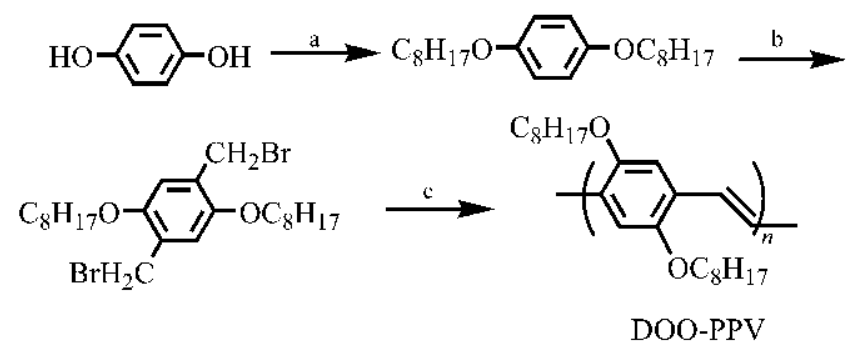

图 1 DOO-PPV 粉末的合成路线

Fig.1 Synthesis route of the DOO-PPV powder

(a) 1-bromooctane, $\mathrm{CH}_{3} \mathrm{CN}$; (b) $\left(\mathrm{CH}_{2} \mathrm{O}\right)_{n}, \mathrm{HBr}$ in acetic acid; (c) potassium tert-butoxide ( $\mathrm{KOBu}$ ), tetrahydrofuran (THF) 
以对苯二酚为原料, 经烷氧基化制备对-辛氧基苯; 再与多聚甲醛、氢澳酸反应获得 2,5-二-辛氧基-1,4二溴甲基苯, 最后利用 Gilch 路线获得红色 DOOPPV 四氢呋喃溶液. 将其滴人大量的甲醇中, 析出 红色絮状的DOO-PPV固体, 过滤后在 $0{ }^{\circ} \mathrm{C}$ 下再溶于 二氯甲烷中, 除去沉淀旋干获得可溶解于普通有机 溶剂(氯仿、四氢呋喃等)的短链DOO-PPV粉末 ${ }^{[14]}$.

DOO-PPV 的凝胶是通过控制叔丁醇钾滴加速 度及单体浓度获得的, 在含有高浓度单体并快速滴 加叔丁醇钾时, 反应开始几分钟内能够观察到红色 块状固体出现, 待冷却、过滤后, 经甲醇、水及沸氯仿 多次洗涤后获得高度物理缠结的 DOO-PPV 凝胶.

利用可溶的短链 DOO-PPV 粉末制备溶液时, 很难获得浓度大于 $1 \mathrm{mg} \cdot \mathrm{mL}^{-1}$ 的溶液, 因此制备了 四氢呋喃和甲苯的 DOO-PPV 饱和溶液, 以石英片 子为基质, 采用 $1000 \mathrm{r} \cdot \mathrm{min}^{-1}$, 旋 $3 \mathrm{~min}$ 即获得相应 的薄膜.

\section{2 结果与讨论}

\section{1 短链 DOO-PPV 粉末及凝胶的结构表征}

图 2 为凝胶和短链DOO-PPV粉末的FT-IR光谱. 图中均未观察到 $\mathrm{C}-\mathrm{Br}$ 的伸缩振动吸收峰 (波数 在 $613 \mathrm{~cm}^{-1}$ 左右), 表明最终产物无论是不溶于任何 溶剂的聚合物凝胶还是可溶于大多数溶剂的短链 DOO-PPV 粉末, 在脱卤化反应过程中脱溴化氢均 较完全. 同时在它们的红外光谱中均能观察到双烷 氧基取代的聚苯撑乙烯全部红外特征吸收峰(比如 $3057 \mathrm{~cm}^{-1}$ 属于苯环上 $\mathrm{C}-\mathrm{H}$ 伸缩振动吸收峰; 966 $\mathrm{cm}^{-1}$ (凝胶)、968 $\mathrm{cm}^{-1}$ (粉末)属于反式 $\mathrm{C}=\mathrm{C}$ 的伸缩 振动吸收峰; $1205 \mathrm{~cm}^{-1}$ (凝胶)、1208 $\mathrm{cm}^{-1}$ (粉末)属于 $=\mathrm{C}-\mathrm{O}-\mathrm{C}$ 的伸缩振动吸收峰等), 这些红外特征

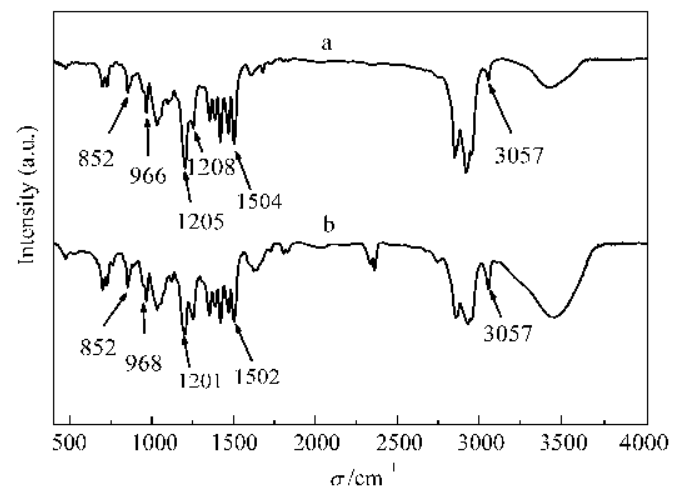

图 2 短链 DO0-PPV 粉末(a)和固态凝胶(b)的 FT-IR 图谱

Fig.2 FT-IR spectra of the short chain DOO-PPV powder (a) and solid gelation (b)
吸收峰与文献报道双烷氧基取代的聚苯撑乙烯的 基本一致 ${ }^{[15]}$. 虽然凝胶和短链DOO-PPV 粉末的红外 吸收峰位置基本一致, 仍然存在微小的差异, 这种微 小的差异可能与分子链的长短及聚集态结构有关.

图 3 为短链 DOO-PPV 粉末和固态凝胶的 FTRaman 光谱. 在它们的拉曼光谱中, $1624 \mathrm{~cm}^{-1}$ (粉末) 和 $1626 \mathrm{~cm}^{-1}$ (凝胶)归属于乙烯基团对称的 $\mathrm{C}=\mathrm{C}$ 伸 缩振动; $1584 \mathrm{~cm}^{-1}$ (粉末) 、 $1586 \mathrm{~cm}^{-1}$ (凝胶) 属于苯环 $\mathrm{C}-\mathrm{C}$ 伸缩振动; $1285 \mathrm{~cm}^{-1}$ (粉末) $1287 \mathrm{~cm}^{-1}$ (凝胶)处 为苯环 $\mathrm{C}=\mathrm{C}$ 伸缩振动; $1115 \mathrm{~cm}^{-1}$ (粉末) 、 $1117 \mathrm{~cm}^{-1}$ (凝胶)为 $\mathrm{C}-\mathrm{C}$ 伸缩和 $\mathrm{C}-\mathrm{H}$ 面内弯曲的混合振动 $\mathrm{g}^{[1,171]}$. 乙烯基团 $\mathrm{C}=\mathrm{C}$ 伸缩振动峰强度与苯环 $\mathrm{C}-\mathrm{C}$ 伸缩 振动峰强度之比与共轭链长度有关 ${ }^{[18]}$. 在短链 DOO-PPV 粉末和固态凝胶的拉曼光谱中, 乙烯基 团 $\mathrm{C}=\mathrm{C}$ 伸缩振动峰强度与苯环 $\mathrm{C}-\mathrm{C}$ 伸缩振动峰 强度之比分别为 0.17 和 0.15 , 表明粉末的共轭主链 短, 这与前面分析结果一致. 通过红外、拉曼光谱分析 表明, DOO-PPV 凝胶与粉末具有相同的化学结构, 在凝胶中并不存在化学交联, 完全是一种物理现象.

\section{2 光谱分析}

图 4 为 DOO-PPV 在不同聚集态的紫外-可见 (UV-Vis)吸收光谱. DOO-PPV 稀溶液的最大吸收峰 为 $453 \mathrm{~nm}$, 与文献报道的基本一致 ${ }^{[19]}$. 固态凝胶的 最大吸收峰在 $500 \mathrm{~nm}$, 并具有较大的半峰宽. 短链 DOO-PPV 粉末的最大吸收峰在 $520 \mathrm{~nm}$ 左右, 其半 峰宽较固态凝胶的半峰宽明显减小, 比溶液的半峰 宽增大. 凝胶中较宽的半峰宽表明, 在凝胶中存在多 种不同长度的 DOO-PPV 分子聚集体. 与凝胶相比, 短链 DOO-PPV 粉末最大吸收峰的红移表示在短链 的 DOO-PPV 粉末中存在较长的分子有序聚集体, 而在凝胶中由于分子的高度链缠结导致其有序区分

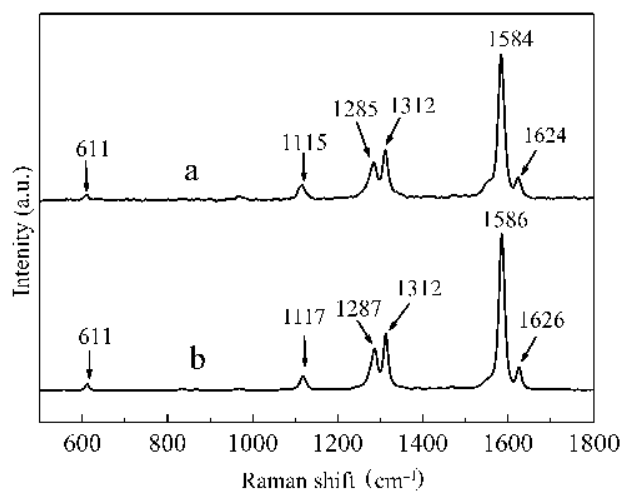

图3 短链D00-PPV 粉末(a)和凝胶 (b)的FT-Raman光谱

Fig.3 FT-Raman spectra of the short chain DOO-PPV powder (a) and solid gelation (b) 


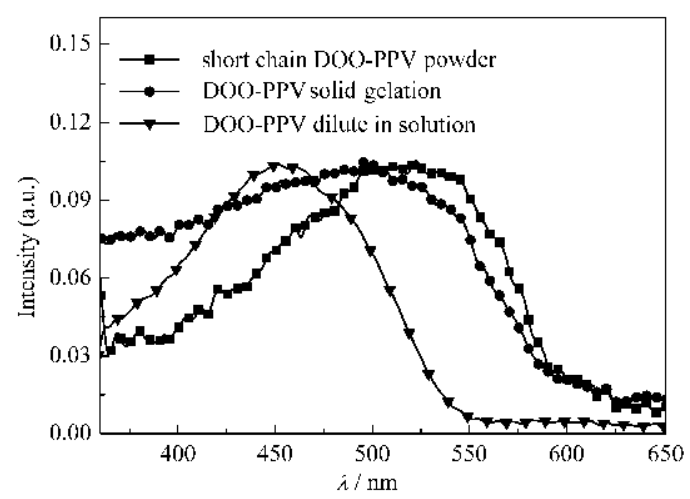

图 4 DOO-PPV 在不同聚集态的紫外-可见吸收光谱

Fig.4 UV-Vis absorption spectra of DOO-PPV with different aggregation

子的长度下降. DOO-PPV 分子在不同聚集态的有 序程度可以被随后的 $\mathrm{X}$ 射线衍射结果所证实.

图 5 为 DOO-PPV 在不同聚集态下的光致发光 (PL)光谱. 在稀的四氢呋喃(THF)溶液中, 最大发射 峰出现在 $547 \mathrm{~nm}$, 属于单链内的发射. 不同 DOOPPV 溶液旋涂的薄膜展示不同的发射光谱, 四氢呋 喃为溶剂制备的薄膜最大发射峰出现在 $568 \mathrm{~nm}$, 肩 峰出现在 $612 \mathrm{~nm}$, 半峰宽为 $50 \mathrm{~nm}$; 由甲苯为溶剂 所获得的薄膜最大发射峰红移到 $572 \mathrm{~nm}$, 肩峰出现 在 $614 \mathrm{~nm}$, 半峰宽为 $42 \mathrm{~nm}$. 这种结果是 DOO-PPV 分子在不同稀溶液中聚合物链不同状态造成的 ${ }^{[20]}$, 通常情况下, 烷氧基取代的聚苯撑乙烯在含苯化合 物中分子链易于舒展, 而在四氢呋喃中分子链更容 易卷曲. 在甲苯为溶剂的薄膜和四氢呋喃为溶剂的

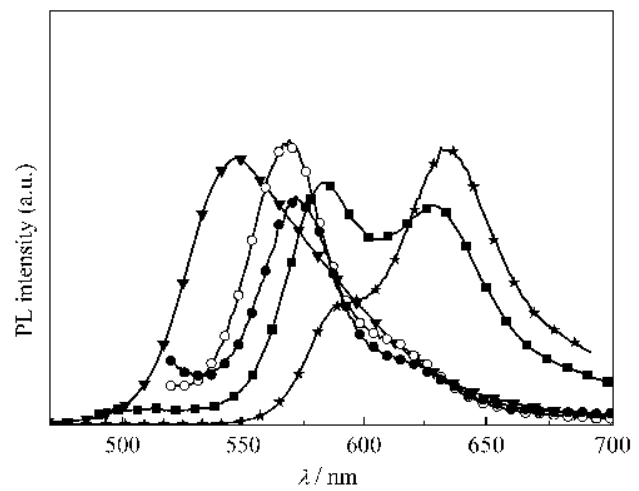

图 5 不同聚集态 DOO-PPV 的光致发光光谱

Fig.5 PL spectra of DOO-PPV in different aggregation

excited wavelength: solution $450 \mathrm{~nm}$, film and powder $500 \mathrm{~nm}$;

- - - short chain DOO-PPV powder;

- - DOO-PPV solid gelation;

- $\mathbf{\nabla}$ - DOO-PPV in diluted solution (THF as solvent);

- - DOO-PPV film (THF as solvent);

- - DOO-PPV film (toluene as solvent)
薄膜中, 短波长发射峰强度与长波长发射峰的强度 之比分别为 3.52 和 3.24 . 在四氢呋喃浓溶液中, 线 型 DOO-PPV 分子已经存在许多链内自聚集和链间 聚集现象, 当在甲醇溶剂中沉淀析出时, 这种状态被 保留下来. 与旋涂薄膜相比, 分子形成固态的时间相 对较长, 有利于不同分子间通过 $\pi-\pi$ 相互作用而形 成较多的有序结构, 因此链间聚集增强, 导致长波长 发射峰强度及半峰宽同时增加, 在短链 DOO-PPV 粉末的苂光光谱中, 在 583 和 $629 \mathrm{~nm}$ 处出现较强 的发射峰, 短波长与长波长发射峰强度之比为 1.10 , 半峰宽为 $91 \mathrm{~nm}$. DOO-PPV 凝胶为链增长过程中由 高度链缠结引起的不溶任何溶剂的固体. 依据 Gilch 路线合成机理可知, 链增长与脱卤反应同时进行, 分 子的高度刚性使得短链长 DOO-PPV 分子内弯曲变 得困难, 容易形成不同链长的有序区, 这种结构的有 序性能够被分子间缠结固定下来, 因此固态凝胶可 能存在更多较长的有序结构, 链间聚集强于链内自 聚集. 在固态的 DOO-PPV 凝胶荧光光谱中, 发射峰 出现在 590 和 $635 \mathrm{~nm}$ 处, 短波长与长波长发射峰 强度之比为 0.47 . 通过上述分析及所观测苂光光谱 变化表明, 在烷氧基取代的聚苯撑乙稀中, 固态下 短波长发射峰与链内自聚集有关, 而长波长发射峰 与链间聚集有关.

\section{$2.3 \mathrm{X}$ 射线衍射图谱分析}

图 6 为短链 DOO-PPV 粉末 (a)、固态凝胶(b)和 薄膜(c)的 X 射线衍射(XRD) 图谱. 从谱(a)中能够清 楚观察到 $2 \theta=5.94^{\circ}, 14.08^{\circ}, 22.66^{\circ}$ 三个 $X$ 射线衍射 特征峰, 其中 $2 \theta=5.94^{\circ}$ 的衍射峰属于线型聚合物从 溶液到固态所形成较短有序结构, 第二个、第三个衍

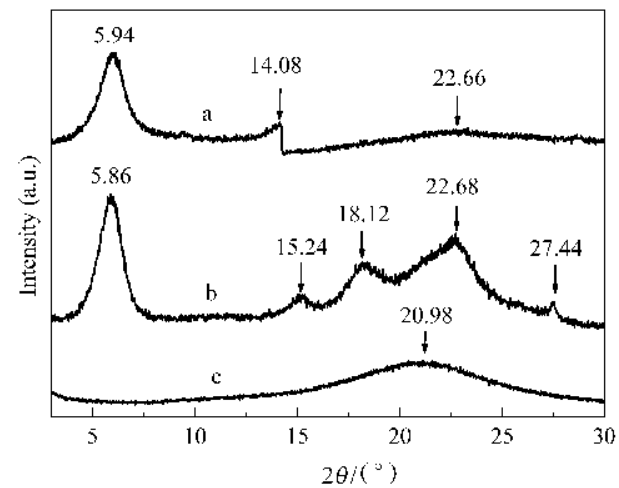

图 6 短链 DOO-PPV 粉末、固态凝胶和薄膜的 $\mathbf{X}$ 射线衍射(XRD)图谱

Fig.6 X-ray diffraction (XRD) patterns of the short chain DOO-PPV powder (a), solid gelation (b) and thin films (c) 
射峰表明在这个状态中存在多个有序结构区 ${ }^{[2]]}$. 从 谱(b) 中可以清楚观察到 $2 \theta=5.86^{\circ}, 15.24^{\circ}, 18.12^{\circ}$, $22.68^{\circ}, 27.44^{\circ}$ 五个 $X$ 射线衍射特征峰, 同样的 $5.86^{\circ} 、 15.24^{\circ} 、 22.68^{\circ}$ 三个 $X$ 射线衍射特征峰位置与 谱(a)接近, 表明可能具有相同形成机制, 其中 $2 \theta=$ $5.86^{\circ}$ 的衍射峰也与聚合物从溶剂中析出时产生的 较小有序结构相关. 令人惊讶的是, 在 $2 \theta=18.12^{\circ}$, $27.44^{\circ}$ 处又增加了两个新 $\mathrm{X}$ 射线衍射特征峰, 表 明固态凝胶中存在更多的不同长度的有序区, 亦表 明不同分子的链间聚集体增多. 谱(c)中仅仅在 $2 \theta=$ $20.98^{\circ}$ 处观察到宽、弱的 X 射线衍射特征峰, 表明薄 膜与粉末及固态凝胶相比, 其有序结构最少.

\section{3 结 论}

合成、表征了具有高度物理缠结的 DOO-PPV 凝胶. 凝胶与短链 DOO-PPV 粉末具有相同的化学 结构, 分子间并不存在化学交联; 在 DOO-PPV 薄 膜、粉末、固态凝胶的苂光光谱中, 短波长发射峰强 度与长波长发射峰强度的比值依次降低; 固态凝胶 中存在更多不同长度的有序结构, 薄膜的有序结构 最少. 在双烷氧基聚苯撑乙烯衍生物的固态光致发 光光谱中, 短波长的发射峰与链内自聚集相关, 而长 波长发射峰与链间聚集有关.

\section{References}

1 Burroughes, J. H.; Bradley, D. D. C.; Brown, A. R. Nature, 1990, 347: 539

2 Liu, J. C.; Wang, W. L.; Yu, H. Z.; Wu, Z. L.; Peng, J. B.; Cao, Y. Solar Energy Materials \& Solar Cells, 2008, 92: 1403

3 Friend, R. H.; Gymer, R. W.; Holmes, A. B. Nature, 1999, 397: 121
4 Gustafsson, G.; Cao, Y.; Treacy, G. M.; Klavetter, F.; Colaneri, N.; Heeger, A. J. Nature, 1992, 357: 477

5 Sariciftci, N. S.; Smilowitz, L.; Heeger, A. J.; Wudl, F. Science, 1992, 258: 1474

6 Nuyen, T. Q.; Martini, I. B.; Liu, J.; Schwartz, B. J. J. Phys. Chem. $B, 2000,104: 237$

7 Schwartz, B. J.; Hide, F.; Andersson, M.; Heeger, A. J. Chem. Phys. Lett., 1997, 265: 327

8 Samuel, I. D. W.; Rumbles, G.; Collison, C. J.; Friend, R. H.; Moratti, S. C.; Holmes, A. B. Synth. Met., 1997, 84: 497

9 Gebler, D. D.; Wang, Y. Z.; Fu, D. K.; Swager, M.; Epstein, A. J. J. Chem. Phys., 1998, 108: 7842

10 Osaheni, J. A.; Jenekhe, S. A. Macromolecules, 1994, 27: 739

11 Chen, Z. K.; Pan, J. Q.; Xiao, Y.; Lee, N. H.; Chua, S. J.; Huang, W. Thin Solid Films, 2000, 363: 98

12 Schwalm, T.; Wiesecke, J.; Immel, S.; Rehahn, M. Macromolecules, 2007, 40: 8842

13 White, J. D.; Hsu, J. H.; Fann, W. S.; Yang, S. C.; Dern, C. R.; Chen, S. A. Chem. Phys. Lett., 2001, 338: 263

14 Johansson, D. M.; Srdanov, G.; Yu, G.; Theander, M.; Lnganäs, O.; Andersson, M. R. Macromolecules, 2000, 33: 2525.

15 Atreya, M.; Li, S.; Kang, E. T.; Neoh, K. G.; Ma, Z. H.; Tan, K. L.; Huang, W. Polymer Degradation and Stability, 1999, 65: 287

16 Yang, S. H.; Nguyen, T. P.; Rendu, P. L.; Hsu, C. S. Thin Solid Films, 2005, 471: 230.

17 That, C. T.; Phillips, M. R.; Nguyen, T. P. Journal of Luminescence, 2008, 128:2031

18 Nguyen, T. P.; Tran, V. H.; Destruel, P.; Oelkrug, D. Synth. Metals, 1999, 101: 633

19 Meyer, R. K.; Benner, R. E.; Vardeny, Z. V.; Liess, M.; Ozaki, M.; Yoshini, K.; Ding, Y.; Barton, T. Synth. Metals, 1997, 84: 549

20 Nguyen, T. Q.; Doan, V.; Schwartz, B. J. J. Chem. Phys., 1999, 110: 4068

21 Breban, L.; Lutsen, L.; Vanhoyland, G.; Haen, J. D.; Manca, J.; Vanderzande, D. Thin Solid Films, 2006, 511-512: 695 